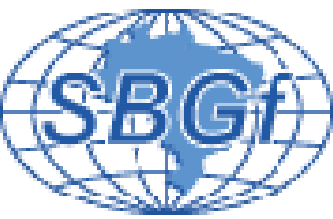

\title{
Modelagem elétrica 2D a partir de dados de eletrorresistividade do antigo Lixão de Ribeirão Preto-SP
}

\author{
Sergio Jr. S. Fachin ${ }^{1}$, Vagner R. Elis ${ }^{2}$, Welitom R. Borges ${ }^{1}$, Alexandre L. Lago ${ }^{1}$ \& Geórgia C. Souza ${ }^{1}$ \\ ${ }^{1}$ Curso de Pós-Graduação em Geofísica. Instituto de Astronomia, Geofísica e Ciências Atmosféricas. \\ ${ }^{2}$ Departamento de Geofísica. Instituto de Astronomia, Geofísica e Ciências Atmosféricas.
}

\section{Copyright 2006, SBGf - Sociedade Brasileira de Geofísica}

This paper was prepared for presentation at the $2^{\text {nd }}$ Symposium Brazilian Geophysical in Natal, Brazil, 21-23 September 2006.

Contents of this paper were reviewed by the Technical Committee of the $2^{\text {nd }}$ Symposium Brazilian Geophysical. Ideas and concepts of the text are authors responsibility and do not necessarily represent any position of the SBGf, its officers or members. Electronic reproduction or storage of any part of this paper for commercial purposes without the written consent of the Brazilian Geophysical Society is prohibited.

\section{Resumo}

Para um planejamento efetivo de aquisições de eletrorresistividade 2D em uma área com informações geológicas e geofísicas, a utilização de modelagens diretas e inversas facilita a melhor escolha dos parâmetros de campo. Neste trabalho realizamos modelagens de um perfil de caminhamento elétrico na área do antigo lixão de Ribeirão Preto-SP utilizando-se dos arranjos dipolo-dipolo, pólo-dipolo e wenner alfa. Os resultados mostraram maior eficiência do arranjo dipolodipolo na identificação da geometria das cavas de resíduos.

\section{Introdução}

Os ensaios geofísicos de eletrorresistividade 2D são utilizados extensamente para identificar e delimitar áreas com geologia complexa. Dentre as técnicas existentes no método da eletroresistividade, a técnica do caminhamento elétrico identifica as variações laterais de resistividade elétrica em subsuperfície (Ward, 1990).

Até década de 90 somente existiam disponíveis no mercado softwares que possibilitavam a interpretação qualitativa de dados de resistividade elétrica. $O$ significante avanço na última década no desenvolvimento de equipamentos de aquisição e nas técnicas de campo proporcionou uma maior aplicação de ensaios de resistividade 2 e também no desenvolvimento de algoritmos de inversão para a interpretação quantitativa de dados (Inman, 1975; Pelton et al., 1978; Loke \& Baker, 1996) permitindo assim, a obtenção de modelos com melhor resolução e maior precisão.

Existem atualmente dois tipos principais de modelagens a Modelagem Direta e a Inversa. A primeira é a simulação do efeito a partir de um modelo físico, ao passo que a segunda objetiva inferir o modelo físico correspondente aos efeitos observados (Duarte, 2003).

A utilização de modelagens diretas no planejamento de aquisições de eletrorresistividade é uma rotina que favorece a escolha dos melhores parâmetros de campo (espaçamento entre os eletrodos, arranjo eletródico, profundidade de investigação e resolução lateral), bem como do dimensionamento dos equipamentos. Este tipo de modelagem é possível quando há informações diretas sobre as características físicas do meio (espessura das camadas geológicas, geometria e profundidade do alvo). Este tipo de modelagem não é uma prática muito convencional no Brasil, pois grande parte das Instituições de Ensino e Pesquisa considera mais importante a experiência dos profissionais nas práticas de campo.

O objetivo principal deste trabalho é mostrar resultados de modelagem direta bidimensional de uma seção geológica-geofísica obtida na área do antigo Lixão de Ribeirão Preto-SP (Figura 1) e verificar o melhor arranjo eletródico que possibilite a reconstrução da geometria das cavas.

Este lixão se caracteriza por apresentar duas cavas alongadas com 300 metros de comprimento por 40 a 60 metros de largura onde os resíduos sólidos urbanos eram dispostos. Esta área, desde 1990, vem sendo estudada através de métodos diretos e indiretos de investigação de subsuperfície (Zuquette et al., 1990; Elis \& Zuquette, 1998; Petrobras/Unaerp, 1997; Elis, 1999; Elis \& Zuquette, 2002; Mondelli, 2004).

\section{Metodologia}

A modelagem 2D foi realizada através do software RES2DMOD (Loke, 2002) que utiliza o método de diferenças finitas. O software divide a subsuperfície numa malha regular (grid) contendo diversos blocos retangulares (Figura 2). O método de diferenças finitas determina o potencial para os nós desta malha regular de blocos retangulares, sendo $L$ os nós na horizontal e $M$ os nós na direção vertical. O software utiliza uma malha de 2 a 4 nós horizontais por espaçamento entre eletrodos. 


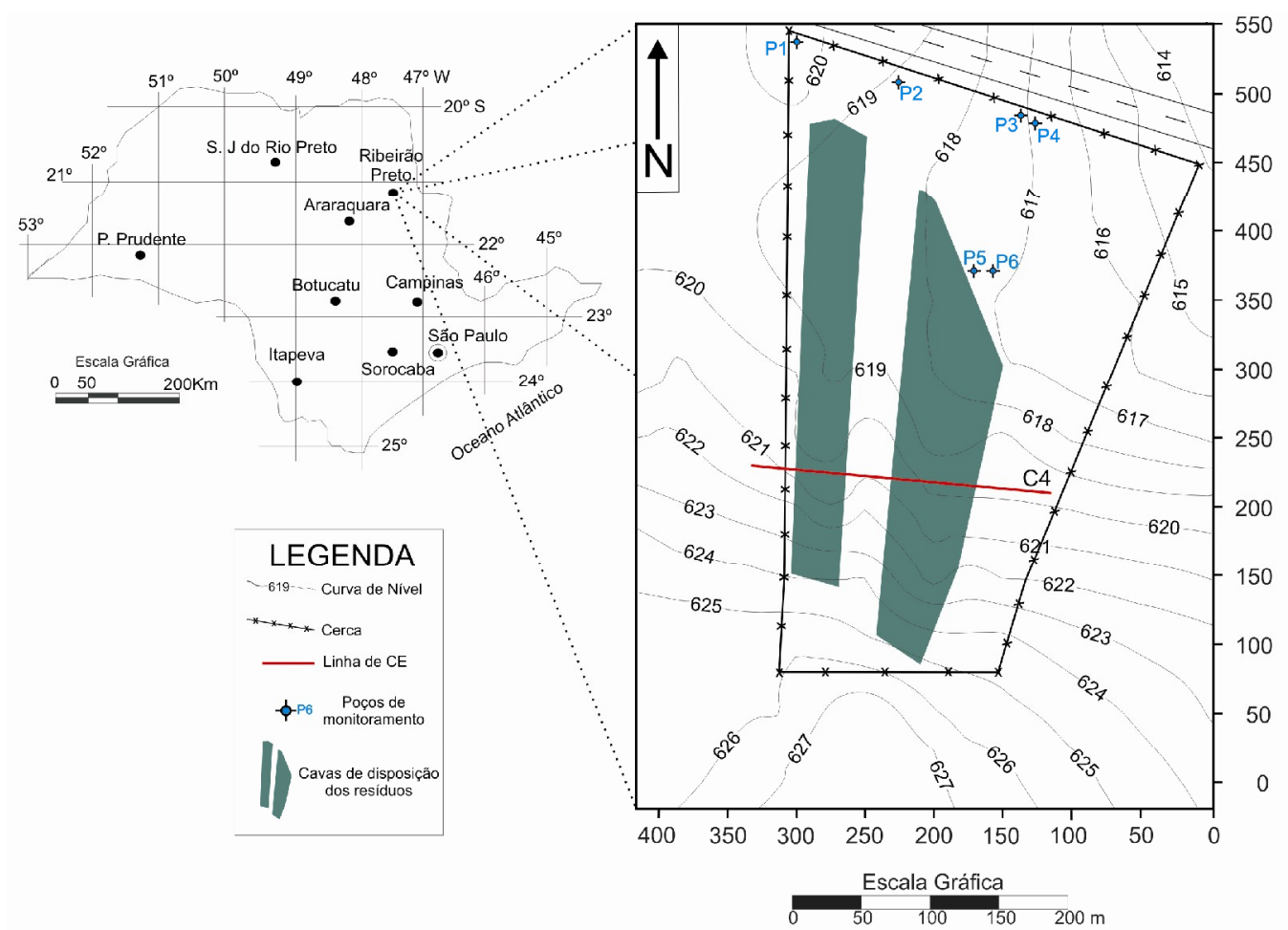

Figura 1 - Mapa de localização da área mostrando a disposição da linha de caminhamento elétrico modelada.

O programa necessita da entrada dos valores de resistividade elétrica para cada bloco retangular do modelo. O modelo geológico-geofísico (Figura 3a), objeto de pesquisa deste trabalho, foi elaborado a partir de dados de resistividade elétrica obtidos no antigo lixão do município de Ribeirão Preto-SP (Elis, 1999).

Após a elaboração do modelo de blocos de resistividade, foram simuladas respostas de aquisições elétricas para os arranjos dipolo-dipolo, pólo-dipólo e wenner alfa (Ward, 1990), com espaçamento entre os eletrodos de 5 metros e 10 níveis de investigação. Os resultados da modelagem direta (Figura 3) foram exportados para o software Res2dinv (Loke, 2004a) com o intuito de verificar qual 0 arranjo que melhor reconstruiria a geometria das cavas. Um ruído gaussiano de $5 \%$ foi adicionado em todas as seções fornecidas pela modelagem direta, para mostrar que o esquema de inversão é razoavelmente robusto e pode trabalhar em ambientes geológicos complexos ou ruído instrumental.

A modelagem inversa com o software Resd2inv foi terminada na terceira iteração devido a utilização de uma versão freeware do programa. Os erros médios quadráticos (RMS - Root Mean Square) obtidos para os modelos inversos dos arranjos eletródicos foram: 9,33\% (dipolo-dipolo); 7,49\% (pólo-dipólo) e 5,73\% (wenner alfa).

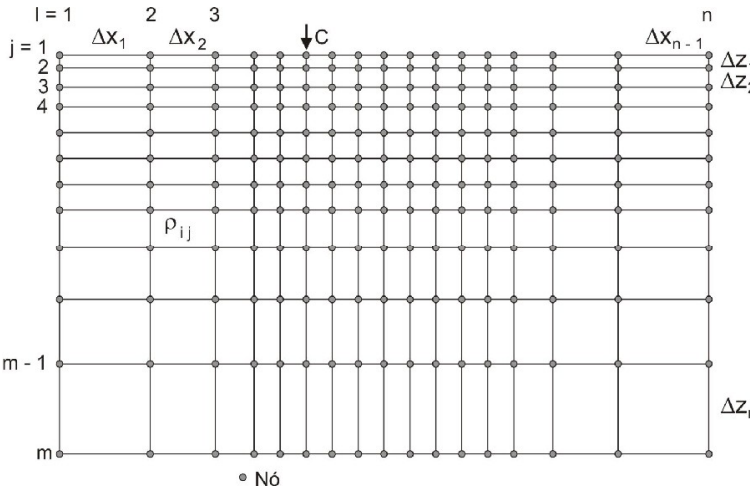

Figura 2 - Diagrama esquemático de malha de elementos finitos utilizada pelo programa RES2Dinv (Loke, 2002).

\section{Resultados e Discussões}


A Figura 3 mostra os resultados da modelagem direta obtidos para os arranjos dipólo-dipólo (Figura 3b), pólodipólo (Figura 3c) e wenner alfa (Figura 3d). Em todos os modelos, houve uma expressiva diminuição na resistividade elétrica sobre as cavas de resíduos. Sendo mais evidente e de maior amplitude no modelo obtido com o arranjo dipolo-dipolo.

Os modelos diretos obtidos com os arranjos dipolo-dipolo e pólo-dipólo sofreram uma forte influência com a variação lateral de resistividade.

Nota-se também que o modelo direto obtido com o arranjo wenner alfa (Figura 3d) mostrou o melhor resultado na reconstrução do modelo geológico-geofísico de entrada (Figura 3a).

A Figura 4 mostra o resultado da modelagem inversa obtidos com os arranjos dipolo-dipolo (Figura 4b), pólodipolo (Figura 4c) e Wenner alfa (Figura 4d). Os resultados obtidos com 0 arranjo dipolo-dipolo conseguiram delimitar com maior definição a geometria das cavas, ou seja, melhor resolução lateral, no entanto, com menor profundidade de investigação. $O$ modelo inverso obtido com o arranjo wenner alfa possibilitou uma maior profundidade de investigação, entretanto, sofreu distorções na base das cavas.

\section{Conclusões}

Os resultados mostram que o arranjo dipolo-dipolo identifica melhor o limite das cavas de resíduos; o arranjo wenner alfa permite, com os dados brutos de campo, uma interpretação preliminar dos dados. Apesar de o arranjo wenner alfa fornecer uma melhor profundidade de investigação, o tempo gasto em campo para mobilização dos eletrodos é muito superior ao do arranjo dipolo-dipolo e do pólo-dipolo.

A modelagem direta mostrou-se uma excelente ferramenta para planejamento de geofísica de campo (arranjo eletródico, espaçamento e profundidade de investigação).

\section{Agradecimentos}

Os autores agradecem a Coordenação de Aperfeiçoamento de Pessoal de Nível Superior (CAPES) pelo financiamento de bolsas de pesquisa. Ao Departamento de Geofísica do IAG/USP pela oportunidade da realização desta pesquisa.

\section{Referências Bibliográficas}

Duarte, O. O., 2003. Dicionário enciclopédico inglêsportuguês de Geofísica e Geologia. 2a Ed. Rio de Janeiro: SBGf. p. 352. Rio de Janeiro, RJ.

Elis, V. R., 1999. Avaliação da aplicabilidade de métodos elétricos de prospecção geofísica no estudo de áreas utilizadas para disposição de resíduos. Tese de
Doutorado, Instituto de Geociências e Ciências Exatas, UNESP, Campus de Rio Claro-SP, 264 p.

Elis, V. R. \& Zuquette, L. V., 1998. Avaliação de contaminação por resíduos de curtume com a utilização de métodos elétricos. Encontro de Geotecnia e Meio Ambiente e II Workshop de Geofísica Aplicada, Rio Claro-SP. CD-ROM, trabalho Elis.doc.

Elis, V. R. \& Zuquette, L. V., 2002. Caracterização geofísica de áreas utilizadas para disposição de resíduos sólidos urbanos. Revista Brasileira de Geociências. São Paulo-SP. Volume 32. 119-134 p.

Inman J., 1975. Resistivity inversion with ridge regression. Geophysics 40, 798-817.

Loke, M. H. \& Barker, R.D., 1996. Rapid least-squares inversion of apparent resistivity pseudosections by a quasi-Newton method. Geophysical Prospecting 44, 131152.

Loke, M. H., 1999. RES2DMOD ver. 2.2, Rapid 2D resistivity forward modelling using the finite difference and finite- element methods. Wenner (alpha, beta, gamma), inline \& equatorial dipole-dipole, polepole, pole-dipole and Wenner- Schlumberger. Austin. USA. 22p.

Loke, M. H., 2004a. Res2Dinv versão 3.54 for Windows 98/Me/2000/NT/XP. Rapid 2D Resistivity \& IP Inversion using the least-squares method. Software Manual. $133 \mathrm{p}$.

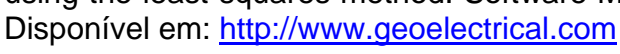

Mondelli, G., 2004. Investigação geoambiental em áreas de resíduos sólidos urbanos utilizando a tecnologia do piezocone. Dissertação (Mestrado). Departamento de Engenharia de Estruturas e Fundações. Escola Politécnica da Universidade de São Paulo. 264 p.

Pelton, W. H., Rijo, L., Swift, C. M., Jr., 1978. Inversion of two-dimensional resistivity and induced-polarization data: Geophysics, 43, 788-803.

Petrobrás/Unaerp, 1997. Lixão de Serrana: Caracterização do problema ambiental e proposição de soluções (Relatório Técnico - Final). 91 p.

Ward, S., 1990. Resistivity and Induced Polarization Methods. Geotechnical and Environmental Geophysics, Volume 1: Review and Tutorial. Society of Exploration Geophysics, Tulsa, OK, p. 147-189.

Zuquette, L. V.; Gandolfi, N. \& Pejon, O. J., 1990. O mapeamento geotécnico na previsão e prevenção de riscos geológicos em áreas urbanas. I Simpósio Latino Americano sobre Risco Geológico Urbano, São PauloSP, pp. 305-315. 


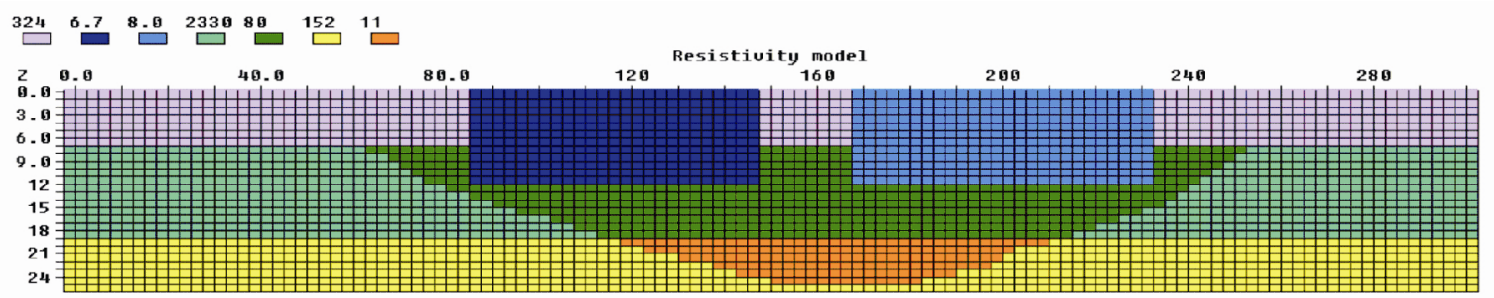

(a) modelo geológico-geofísico
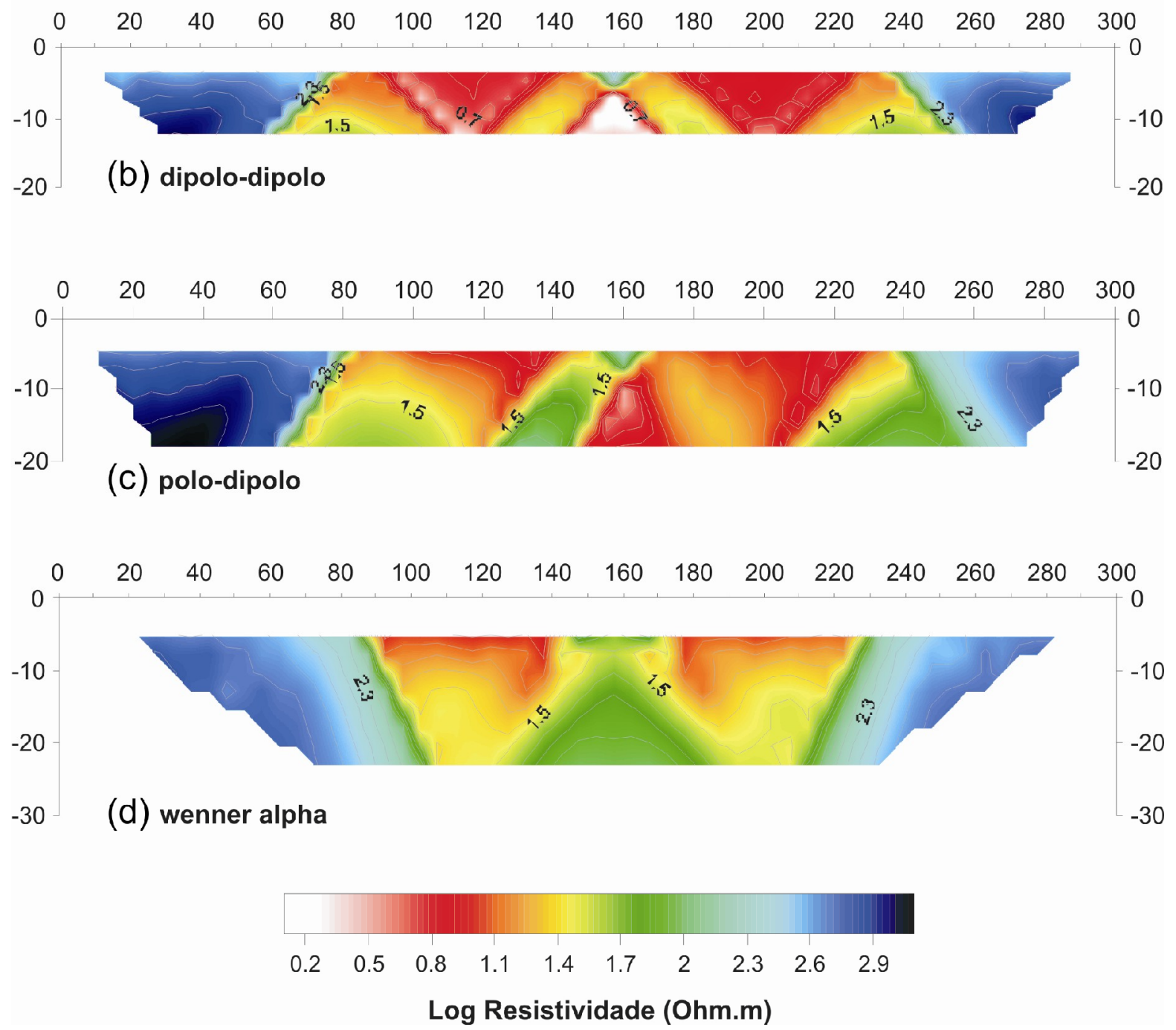

Figura 3 - Modelos diretos de resistividade elétrica. (a) modelo de blocos de resistividade; (b) modelo com arranjo dipolo-dipolo; (c) modelo com arranjo polo-dipolo; (d) modelo com arranjo wenner alfa. 


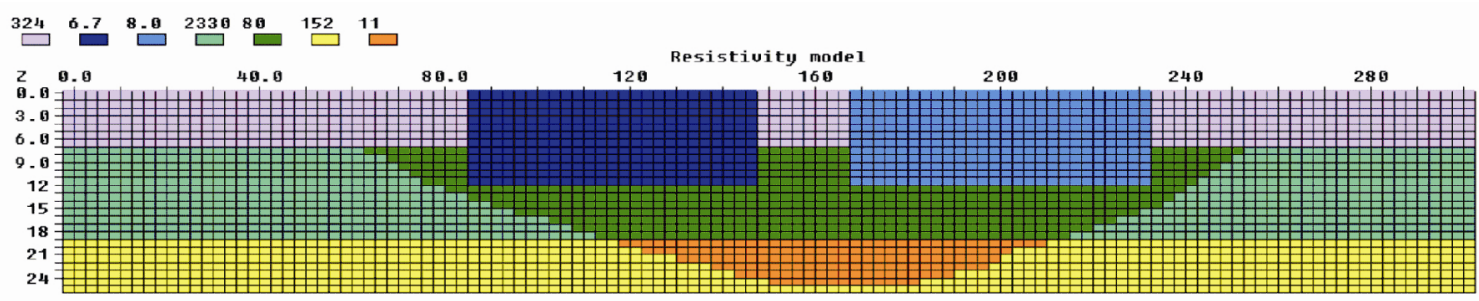

(a) modelo geológico-geofísico
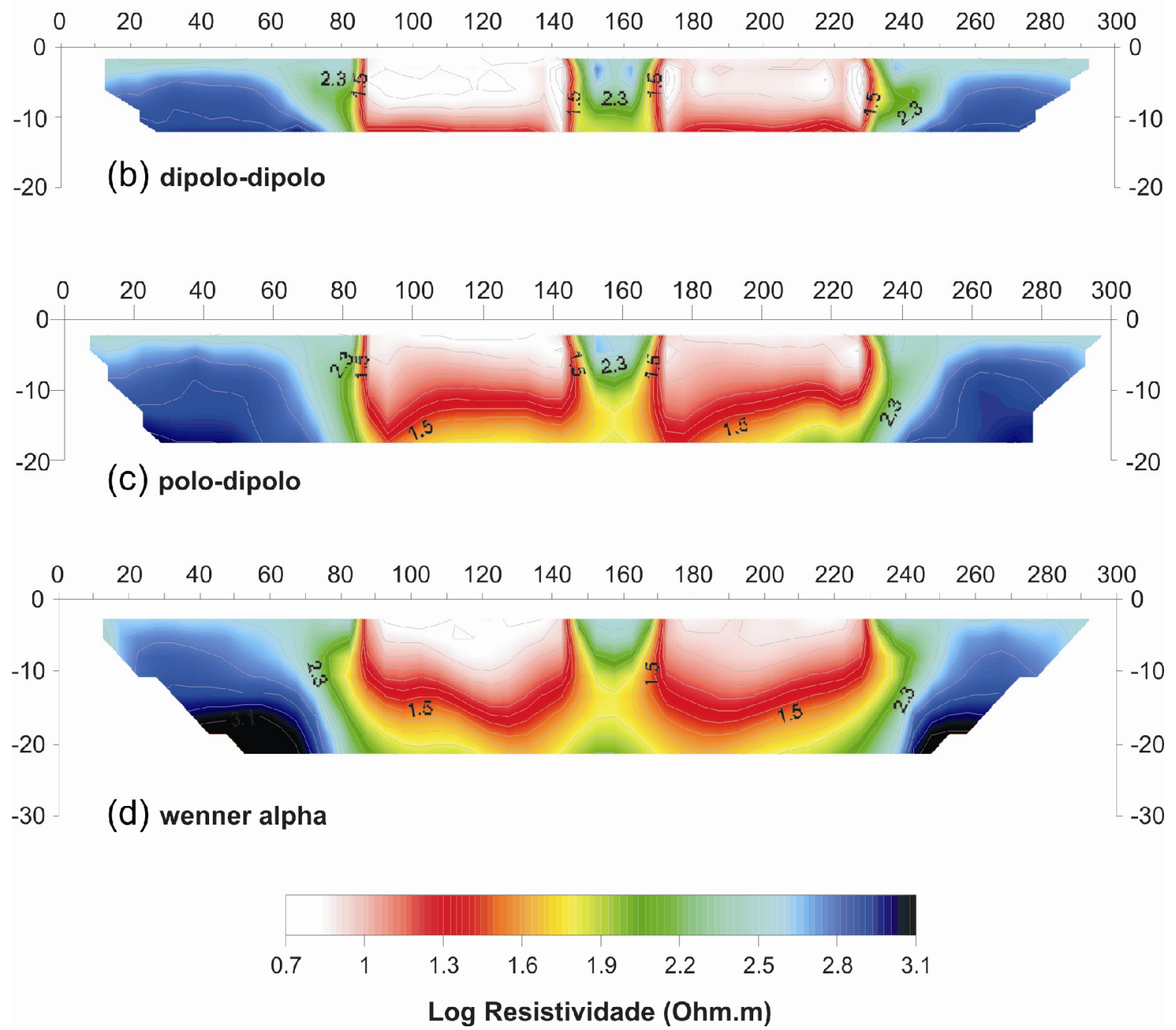

Figura 4 - Modelos inversos de resistividade elétrica. (a) modelo de blocos de resistividade; (b) modelo com arranjo dipolo-dipolo; (c) modelo com arranjo polo-dipolo; (d) modelo com arranjo wenner alfa. 